The Impact of Large-Scale Surveys on Pulsating Star Research

ASP Conference Series, Vol. 203, 2000

L. Szabados \& D. W. Kurtz, eds.

\title{
Nonlinear Pulsations of Convective Stellar Models
}

\section{J. Robert Buchler}

Physics Department, University of Florida, Gainesville, FL 32611, USA

\begin{abstract}
We review the numerical modeling of the nonlinear pulsations of classical variable stars with hydro-codes that include the effects of turbulent convection. Despite their simplicity these turbulent convective recipes appear to remove many of the difficulties that radiative codes faced. In particular, the numerical modeling of double mode pulsations has become possible.
\end{abstract}

\section{Introduction}

Richard Feynman is said to have once remarked something to the effect "now that we have solved the problem of quantum electrodynamics, we will have to solve the real hard problems such as how water flows in a pipe". The stellar problem, because of convection on top of turbulence and the compressibility of the fluid, is even harder to tackle, and several generations of astrophysicists have tried to come to terms with this problem. Turbulence and convection (TC) are necessarily 3 dimensional phenomena, and with the development of faster computers increasingly realistic numerical simulations are being made, although it will be a long time before their spatial resolution approaches that required by the large stellar Rayleigh and small Prandtl numbers. In the meantime stellar physicists continue to attempt to reduce $\mathrm{TC}$ to a $1 \mathrm{D}$ recipe and thus to a mere subroutine that can be used in stellar evolution or pulsation calculations (for a recent update on astrophysical convection cf., e.g., Buchler \& Kandrup 2000). In this paper we review some interesting recent developments in nonlinear pulsation calculations.

The seminal and most influential work has been the mixing length theory (MLT) of Erika Böhm-Vitense (cf. Cox \& Giuli 1968), and many of the newer recipes are extensions of MLT. In its original form it consists of an instantaneous, local approximation in which the convective flux is proportional to the $3 / 2$ power of the convectively unstable entropy gradient, $F_{\mathrm{c}} \propto(-d s / d r)^{3 / 2}$.

The TC recipe that works best in stellar evolution is not necessarily the best for stellar pulsations. Indeed, in stellar evolution the convective time scales are typically many orders of magnitude smaller than the evolutionary time scales. Furthermore, convective overshooting is very important because it mixes the chemical elements with often drastic consequences for nuclear burning and the subsequent evolution. In contrast, mixing plays no role in stellar pulsation because the pulsating envelopes are chemically homogeneous. But here we have large velocity fields and shear motions, so that time dependence of TC may have to be taken into account. Thus the pulsation time scales, while generally 
longer than the convective time scales, are sufficiently close that there can be a feedback between pulsation and convection. This feedback is further enhanced because the convective regions that are caused by large opacity are also regions where pulsational driving occurs. An illustration of the time dependence of the turbulent energy and convective flux during a pulsational cycle has been presented in Buchler et al. (1999, [BYKG99], Figs. 1 and 2).

How good are MLT and its extensions? An important 3D simulation by Cattaneo, Brummell, \& Toomre (1991) indicated first, that the convective flow is dominated by large downflows, but that these flows are 'convectively neutral' in the sense that they carry as much kinetic energy downward as enthalpy upward, and second, that the convective flux is dominated by small scale upflows, precisely the type of picture that underlies MLT. However, for computational reasons, the Prandtl numbers used in the calculations were orders of magnitude larger than the stellar ones, and the Rayleigh numbers orders of magnitude smaller. Furthermore, the boundary conditions were fixed, whereas in a star convection has to adjust itself so that together with radiation it carries the given total energy flux (in a static context).

We recall the hydrodynamic equations in the context of radial stellar pulsation:

$$
\begin{aligned}
\frac{d u}{d t} & =-\frac{1}{\rho} \frac{\partial}{\partial r}\left(p+p_{\mathrm{t}}+p_{\nu}\right)-\frac{G M}{r^{2}} \\
\frac{d e}{d t}+p \frac{d v}{d t} & =-\frac{1}{\rho r^{2}} \frac{\partial}{\partial r}\left[r^{2}\left(F_{\mathrm{r}}+F_{\mathrm{c}}\right)\right]-\mathcal{C} .
\end{aligned}
$$

For the hydrodynamics all we need is a recipe for the turbulent pressure $p_{\mathrm{t}}$, the eddy viscous pressure $p_{\nu}$, the convective flux $F_{\mathrm{c}}$, and the source and sink of turbulent energy $\mathcal{C}$.

\section{The Turbulent Convective Model Equations}

Many recipes have been suggested to compute these four quantities. A very nice, albeit dated, review is that of Baker (1987), and for an update see Giménez, Guinan, \& Montesinos (1999). Many of these are far too complicated (e.g. up to 10 nonlinear PDEs) and numerically tricky to implement in hydro-codes. Since this review concerns primarily stellar pulsations with an emphasis on nonlinear calculations, we will limit ourselves to mentioning the time-dependent recipes that have actually been used in nonlinear hydrodynamics calculations. All these recipes involve the addition of a single time-dependent diffusion equation for the turbulent energy $e_{\mathrm{t}}$ :

$$
\frac{d e_{\mathrm{t}}}{d t}+\left(p_{\mathrm{t}}+p_{\nu}\right) \frac{d v}{d t}=-\frac{1}{\rho r^{2}} \frac{\partial}{\partial r}\left(r^{2} F_{\mathrm{t}}\right)+\mathcal{C} .
$$

The ancillary, defining equations are 


$$
\begin{aligned}
\mathcal{C} & =\mathcal{S}-\epsilon \\
\epsilon & =\alpha_{\mathrm{d}} e_{\mathrm{t}}^{3 / 2} / \Lambda \\
\Lambda & =\alpha_{\Lambda} H_{\mathrm{p}} \\
H_{\mathrm{p}} & =(d \ln p / d r)^{-1}=p /(\rho g) \\
p_{\mathrm{t}} & =\alpha_{\mathrm{p}} \rho e_{\mathrm{t}} \\
p_{\nu} & =-\alpha_{\nu} \Lambda \rho e_{\mathrm{t}}^{1 / 2} r(\partial(u / r) / \partial r) \\
F_{\mathrm{t}} & =-\alpha_{\mathrm{t}} \alpha_{\Lambda} \rho H_{\mathrm{p}} e_{\mathrm{t}}^{1 / 2}\left(\partial e_{\mathrm{t}} / \partial r\right) .
\end{aligned}
$$

For the sake of simplicity these equations disregard some features of convection and therefore have their shortcomings. They are based on a diffusion approximation $\left(F_{\mathrm{c}} \propto d s / d r\right.$ and $F_{\mathrm{t}} \propto d e_{\mathrm{t}} / d r$ ) and ignore nondiffusive transport, e.g. by plumes. They also disregard pressure fluctuations and are limited to subsonic convective velocities. Radiative losses in the convection, however, can easily be incorporated (Wuchterl \& Feuchtinger 1998; Buchler \& Kolláth 2000 [BK00]). It is only a comparison with observational constraints that will ultimately decide on the quality of the approximations in the context of stellar pulsations.

The recipes that have been used in the nonlinear codes fall into three groups depending on the choice of the functional relationships of $F_{\mathrm{c}}$ and $\mathcal{C}$ on $e_{\mathrm{t}}$ and the dimensionless entropy gradient which we call $\mathcal{Y} \equiv\left(H_{\mathrm{p}} / c_{\mathrm{p}}\right) d s / d r$ (not to be confused with the helium abundance). We refer to BK00 for further details. They are: 1) the Stellingwerf (1982) [S] formulation, used by the Italian group (Bono \& Stellingwerf 1994; Bono et al. 1997 [BCCM97]) and by Gehmeyr (1992), 2) the Kuhfuß (1986) [K] formulation (cf. also Gehmeyr \& Winkler 1992) used in the Vienna code (Feuchtinger 1998, 1999a [F99a]), and 3) a hybrid Florida [FL] formulation (Yecko, Kolláth, \& Buchler 1998 [YKB98]) that has been used by Kolláth et al. (1998 [KBBY98]). The Florida hydro-code has recently been extended to run with all three schemes (see below), and, importantly, also to perform a linear stability analysis (linear periods and growth-rates).

$$
\begin{array}{lll}
F_{\mathrm{c}}=A / B & e_{\mathrm{t}}(\mathcal{Y})^{1 / 2} \\
\mathcal{S}=\alpha_{\mathrm{d}} B / \Lambda & e_{\mathrm{t}}(\mathcal{Y})^{1 / 2} \\
F_{\mathrm{c}}=A & e_{\mathrm{t}}^{1 / 2} \mathcal{Y} \\
\mathcal{S}=\alpha_{\mathrm{d}} B^{2} / \Lambda & e_{\mathrm{t}}^{1 / 2} \mathcal{Y} \\
F_{\mathrm{c}}=A & e_{\mathrm{t}}^{1 / 2} \mathcal{Y} \\
\mathcal{S}=\alpha_{\mathrm{d}} B / \Lambda & e_{\mathrm{t}}(\mathcal{Y})^{1 / 2}
\end{array}
$$

where

$$
\begin{aligned}
A & =\alpha_{\mathrm{c}} \alpha_{\Lambda} \rho c_{\mathrm{p}} T \\
B & =\alpha_{\mathrm{s}} \alpha_{\Lambda} \sqrt{\rho \beta T / \rho}=\alpha_{\mathrm{s}} \alpha_{\Lambda} \sqrt{c_{\mathrm{p}} T \nabla_{\mathrm{a}}}=\alpha_{\mathrm{s}} \alpha_{\Lambda} \sqrt{\beta T / \Gamma_{1}} c_{\mathrm{s}} \\
\beta & =(\partial \ln v / \partial T)_{\mathrm{p}}
\end{aligned}
$$



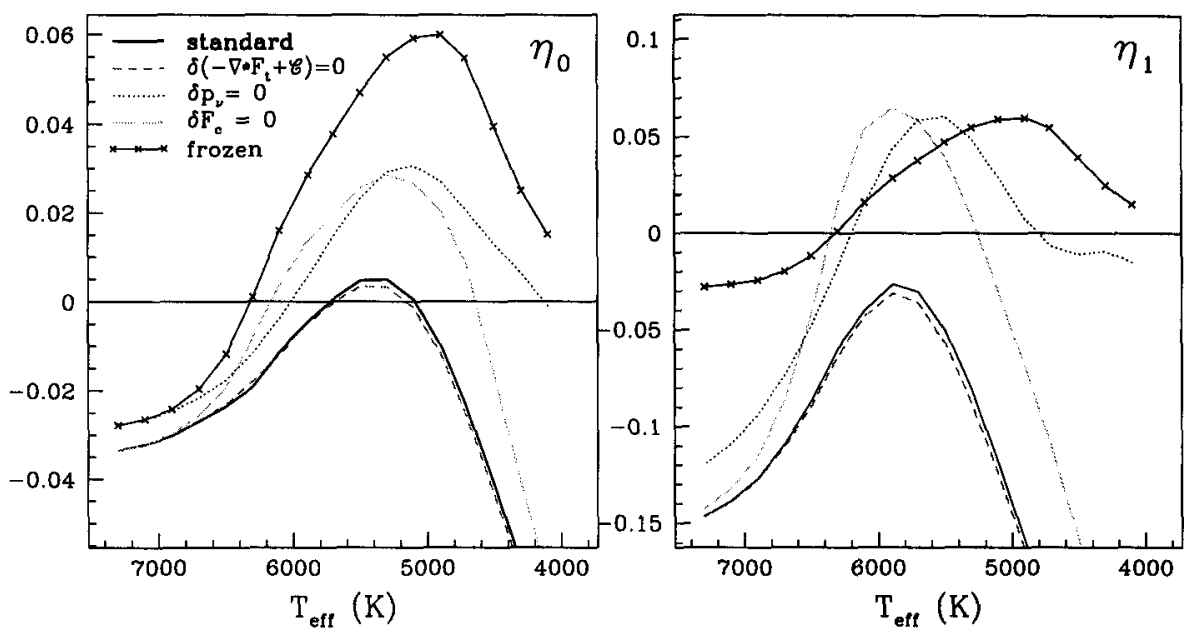

Figure 1. Linear stability of sequence of models. Comparison of different approximations with exact results. Left: fundamental mode, right: first overtone mode.

The recipes involve a total of 7 dimensionless $\alpha$ parameters that are of order unity, but for which theory gives little guidance. They ultimately have to be calibrated by comparing the numerical results to the stellar observations.

The three schemes have been compared in BK00. In the stationary limit and with the disregard of overshooting (no $F_{\mathbf{t}}$ ) eq. 3 reduces to $\mathcal{C}=0$. In other words, it gives an expression for $e_{\mathrm{t}}$ in terms of the local, instantaneous physical quantities such as density and temperature and their gradients, and the ancillary equation 6 provides the convective flux. This is then equivalent to standard MLT. However, in standard MLT the $p_{\mathrm{t}}$ and $p_{\nu}$ are omitted (which is not a good approximation; see below), although they could be readily included once $e_{t}$ is known.

In the time-dependent context one might expect the three formulations to have a very different behavior. However, as shown in BK00, the growth-rates differ very little, and furthermore the three recipes give essentially the same limit cycles as well (Figs. 3 and 6 of BK00).

From these albeit limited comparisons, one is tempted to conclude that most of the differences between the published nonlinear results have more to do with the choice of the $\alpha$ parameters ${ }^{1}$ than with the choice of the time-dependent diffusion equation for the turbulent energy.

\footnotetext{
1 and some modifications such as flux limiters (Wuchterl \& Feuchtinger 1998), small Péclet
} number corrections (BK00), or sonic dissipation (Gehmeyer \& Winkler 1992). 


\section{Linear Stability Properties}

In evolutionary computations it is often customary to compute also the linear stability of the evolving models to delineate the instability strips. For expediency several approximations are often made. We want to stress again (YKB98, BYKG98) that some of these approximations are not very good, as we go on to show in Fig. 1. From right to left we display the normalized growth-rates $\eta=2 \kappa P$ of the fundamental and first overtone, respectively, for a sequence of Cepheid models $\left(M=5 M_{\odot}, L=2060 L_{\odot}, Z=0.02\right)$ as a function of $T_{\text {eff }}(\kappa$ is the growth-rate and $\mathrm{P}$ the period.)

The solid lines denote the nonadiabatic growth-rates, consistent with the hydro and TC equations. The crossed solid lines correspond to the frequently used frozen flux approximation: the MLT flux is included in the computation of the static equilibrium model, but it is ignored in the computation of the linear eigenvalues, and the eddy viscous pressure is also disregarded. Clearly this approximation misses even the blue edge by $\approx 600 \mathrm{~K}$ for the $\mathrm{F}$ mode and indicates instability for the $\mathrm{O} 1$ mode which is solidly stable. In the next two approximations everything is linearized correctly except 1) the perturbation of the turbulent pressure is disregarded (dotted line), and 2) the perturbation of the convective flux is disregarded (long-dashed line) - neither of these a good approximation. The best 'simple' approximation is (dashed line): MLT expression for the flux (derived from $\mathcal{C}-\nabla \cdot F_{\mathrm{t}}=0$ ), its linearization and inclusion of the eddy viscous pressure. Of course, it is also important to choose 'proper' values of the $\alpha$ parameters. A survey of the model behavior makes it quite clear that both a convective flux and a turbulent pressure are needed if one wants to get a reasonable IS. This importance of the eddy viscous pressure was already pointed out a dozen years ago by Baker (1987).

In addition, it should be remembered that nonlinear effects can shift the linear IS boundaries by a few hundred degrees (cf. Fig. 2 below).

\section{RR Lyrae Models, RRab and RRc}

There have been several recent large surveys of nonlinear pulsations of single mode RR Lyrae stars, both fundamental and first overtone pulsations by Bono et al. [BCCM97] and by Feuchtinger (1999b) [F99b]. F99b also compares these results to each other and to the available observations. He finds that the lightcurve Fourier decomposition coefficients of both calculations agree fairly well with Observations, but that there are discrepancies both between the two calculations, and with observations in the low temperature models, i.e. in the most convective models. The pulsation amplitudes of both calculations agree well with observations. (It should be noted though that this is not as stringent a test as the Fourier parameters).

As far as the shapes of the light curves are concerned, BCCM97 obtain sharp, but unobserved spikes (cf. their Figs. 2 and 4). F99b (cf. also Wuchterl \& Feuchtinger 1998) shows that these spikes are due to the fact that the convective flux becomes larger than its physically allowed upper limit, viz. $F_{\mathrm{c}}<\rho c_{\mathrm{p}} T u_{\mathrm{conv}}$. This is a result of the breakdown of the diffusion approximation that is inherent in the TC equations. They propose the introduction of a 'flux limiter' to prevent 
the flux from exceeding this upper limit. As a result F99b obtains light curves that look much more like the observed ones.

The RR Lyrae light-curves obtained by the Florida group do not have any unphysical spikes either, despite the fact that no flux limiter has been used. Feuchtinger (private communication) has traced the absence of spikes to a different choice of $\alpha$ parameters, those of the FL group giving rise to lower overall turbulent energies and velocities.

The computed radial velocity curves do not agree very well with the observations (F99b). It seems that a more thorough calibration of the $\alpha$ parameters is required before the final word is in on whether such a simple 1D TC equation is capable of capturing the essence of convection.

We also note that there are further constraints on the alphas that need to be taken into account. For example, the observed temperature (color) variations can be taken into account. Second, the double-mode RR Lyrae (RRd) stars impose a number of sensitive additional constraints, namely the range of periods and of temperatures over which they occur, as well as the values of the component amplitudes. Finally, we recall that the very frequent Blazhko amplitude modulations have not yet been satisfactorily explained, but that they are likely to also add constraints.

We note a propos the Blazhko effect that a possible mechanism for this effect could be an interaction between pulsation and convection. This becomes particularly favored if normally real and stable convective (diffusion) modes become oscillatory. If these additional vibrational modes are only mildly stable, and if their frequencies are in an $n: 1$ resonance with the excited pulsational mode, then a resonance condition could cause the convective mode to interact nonlinearly with the pulsation and lead to amplitude modulations. We have checked on realistic stellar models that convective modes can indeed become oscillatory, but we had to increase the time scale for convection by a very large factor, and no nonlinear computations have yet been performed.

\section{Morphology of the Instability Strip - Modal Selection}

On the observational side the microlensing projects have produced global pictures of the Cepheid instability strips for the SMC that are absolutely stunning (Udalski et al. 1999; Beaulieu et al. 1995). On the theoretical side, the turbulent convective hydrodynamics codes have shed new light on the problem of modal selection in both Cepheids and RR Lyrae stars. Simultaneously, but totally independently, Feuchtinger (1998) and KBBY98 found RR Lyrae models and Cepheid models, respectively, that pulsated in the fundamental and first overtone modes simultaneously, with stable, and steady amplitudes (i.e. the models were NOT switching modes).

On the basis of these computations (cf. BYKG 99 and Kolláth (2000)) we can infer the following (schematic) Cepheid instability strips (IS) in an HR diagram as shown in Fig. 2. The left subfigure depicts a linear IS. The first overtone IS in the form of a sugarloaf becomes stable above a certain luminosity (and mass). For simplicity we have omitted the second overtone from the picture.

Of course, nonlinear effects change the domain in which the corresponding limit cycles are stable. The right-hand subfigure depicts a schematic of the 


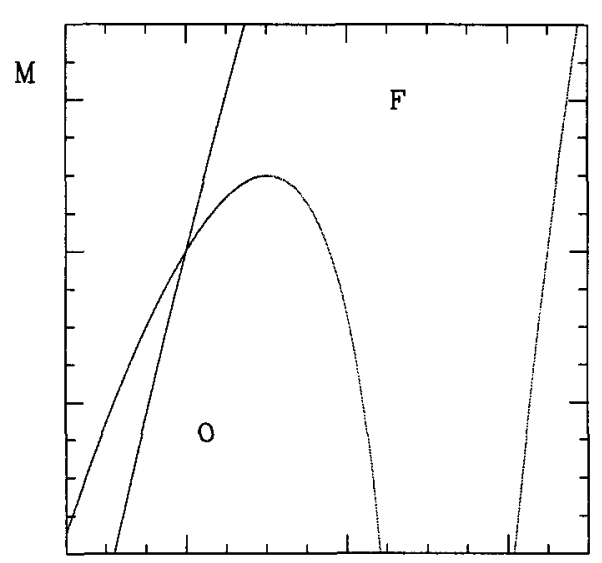

$\mathrm{B}-\mathrm{V}$

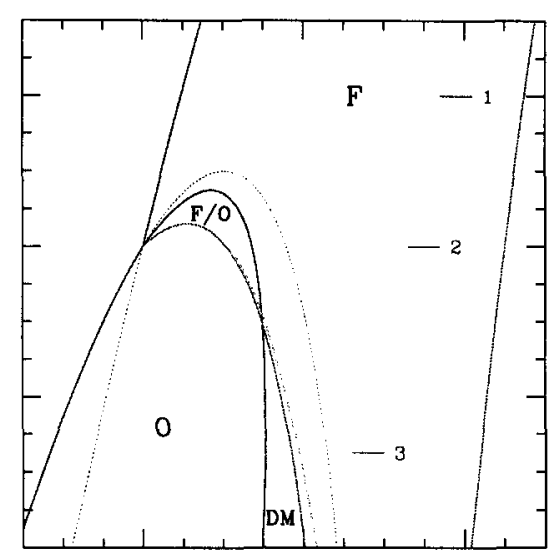

$\mathrm{B}-\mathrm{V}$

Figure 2. Schematic Cepheid instability strip, left: linear, right: nonlinear; cf. text.

nonlinear Cepheid instability diagram. The lines from the left side subfigure that are shown as solid lines are the blue and red boundaries of the fundamental $(\mathrm{F})$ and first overtone $(\mathrm{O})$ ISs. (The now irrelevant parts of the previously shown linear edges are shown as thin dotted lines.) The new additional solid lines are the nonlinear blue and red edges for the fundamental and first overtone modes. Thus the overtone red edge is shifted somewhat to the left, but the fundamental blue edge can be shifted substantially to the right.

Double-mode behavior occurs in the lower wedge-shaped region, delineated on the left by overtone red edge and on the right by a dashed line. Either fundamental or first overtone ( $\mathrm{F} / \mathrm{O}$ ) behavior occurs in the higher luminosity region that is shown as dotted. There may be a narrow region at the interface of the DM and $\mathrm{F}$ regimes in which either DM or fundamental behavior can occur. ${ }^{2}$ We note that a good global understanding of all these regimes can be obtained with the help of the amplitude equation formalism (e.g. BYKG99).

More specifically, for example at level 1 (high luminosity) in Fig. 2 only $\mathrm{F}$ pulsations can occur. At level 2 we have, going from high to low $T_{\text {eff }}$, a regime first overtone pulsations, then a regime of either $\mathrm{O}$ or $\mathrm{F}$ pulsations (hysteresis), and then F only pulsations. At low luminosities, level 3 , there is first a regime of $\mathrm{O}$ only, then of DM only, with possibly a narrow regime of either DM or F, followed by $\mathrm{F}$ only pulsations.

The hydrodynamic calculations indicate that DM behavior occurs only at luminosities that can be noticeably lower than the tip of the overtone instability strip. This is in agreement with the SMC observations (Fig. 5 of Udalski et al. 1999) which show a higher luminosity regime in which both $F$ and $O$ Cepheids

${ }^{2}$ In some computations, with different $\alpha$ parameters, slightly more complicated interfaces have also been obtained. 
occur, and a lower luminosity one in which the DM Cepheids lie (with the exception of a single star).

The RR Lyrae stars, at least within a given cluster, have essentially the same luminosity, mass and composition. The modal selection diagram is therefore essentially the same as for a narrow horizontal strip in the lower part of Fig. 2.

\section{Classical Cepheid Pulsations}

The classical Cepheids are much more diverse than the RR Lyrae stars. They span a wide range of masses, luminosities and metallicities. There are a great deal more observational constraints as well many of which have been summarized in BK00.

For example, the overtone Cepheids have a maximum period $P_{1}^{\max }$ which occurs at the high luminosity tip of the IS. Next, resonances play an important role, viz. a $P_{2} / P_{0}=1 / 2$ resonance at about $10 \mathrm{~d}$ and a $P_{4} / P_{1}=1 / 2$ resonance around $3-4.5 \mathrm{~d}$. This is evidenced by the structure of the Fourier decomposition coefficients of the light and radial velocity curves. We note that in principle it is possible to obtain a purely 'pulsational' mass-luminosity relation by taking advantage of these two resonances. The periods and $T_{\text {eff }}$ at which DM behavior can occur, as well as the $\mathrm{F} / \mathrm{O}$, respectively $\mathrm{O}_{2} / \mathrm{O}_{1}$ amplitude ratios add a very tight constraint as well.

Radiative Cepheid models were found wanting in many respects, besides the obvious one of not providing a red edge (for a review cf. Buchler 1998). In particular the discrepancies are largest for the low $Z$ models for which the linear growth-rates and consequently the pulsation amplitudes are much too large. The resonance masses are also much too small to agree with stellar evolution calculations as was discussed in Buchler et al. (1996). The question therefore arises whether convection can provide a differentially stronger dissipation for the low $Z$ models.

There have been a number of Cepheid computations by the Italian, Vienna and Florida groups, but no comprehensive calculations have yet been made to see if all observational constraints can be simultaneously satisfied. However, there is some good news. For example, with the convective hydro-codes it seems possible to improve the light and radial velocity curves, and in particular to obtain the wide excursion in the observed $\phi_{21}$ Fourier phase of the overtone Cepheids in which purely radiative models had failed. The most dramatic achievement though is the modeling of DM behavior in beat Cepheids (KBBY98).

Interestingly, despite seven adjustable $\alpha$ parameters, it was not possible to impose both the observed upper limit for the period of the first overtone Cepheids and obtain a reasonable width of the fundamental instability strip! This problem was solved when we included the physically required correction for inefficient convection (small Péclet number) (BK00), but at the expense of an additional, eighth free $\alpha$ parameter.

Some properties, such as period-radius relations seem relatively insensitive to the values of the alphas. In Fig. 3 we show the $P-R$ relation that we obtain for Galactic Cepheid models, compared to the observational data. A very similar agreement has been obtained by Bono et al. (1999). 
Observations show that the SMC, LMC and Galactic Cepheids are remarkably similar. For example, the maximum first overtone periods lie around $6 \mathrm{~d}^{3}$ They have approximately the same luminosity, the same pulsation amplitudes, the same Fourier decomposition coefficients, and the dominant $\mathrm{F}$ and $\mathrm{O} 1$ resonances are almost in the same place, i.e. near $10 \mathrm{~d}$ and $3-4.5 \mathrm{~d}$, respectively.

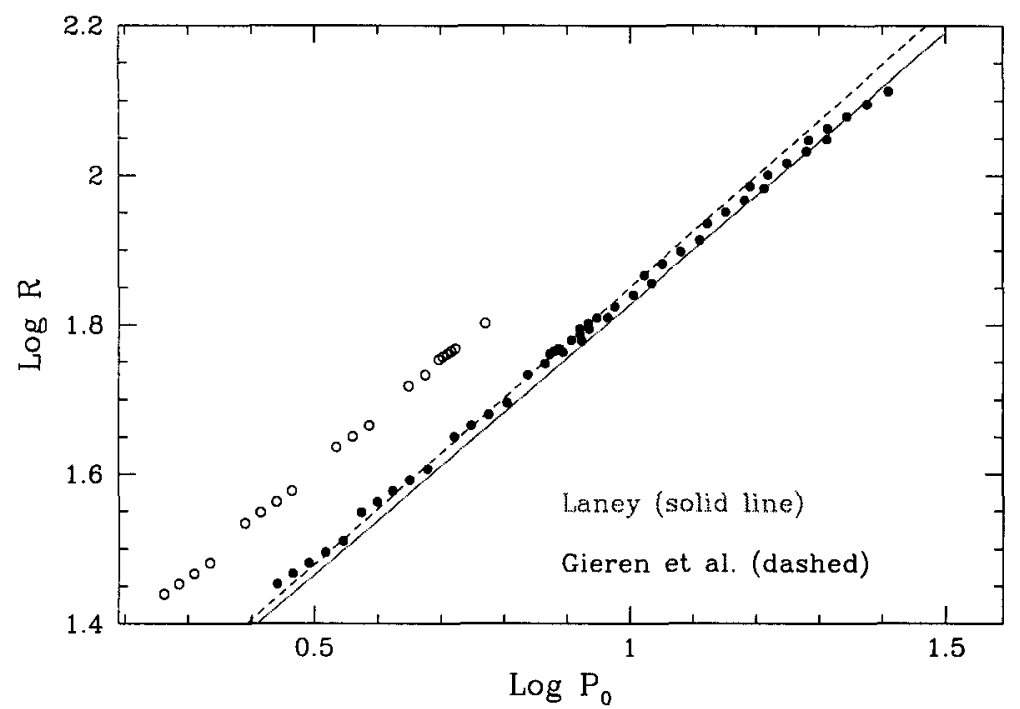

Figure 3. Period-radius relation for Galactic Cepheids. Theoretical convective Cepheid models: fundamental - solid dots, first overtones open circles; Baade-Wesselink radii: Laney (1997) - solid line, Gieren, Fouqué, \& Gómez (1997) - dashed line.

The not-so-good news is that at this time it does not seem possible to obtain good models both for the Galactic, and for the low $Z$ Magellanic Cloud Cepheids with the same calibration of the eight $\alpha$ parameters. Turbulent convection does not provide larger dissipation for the low $Z$ models. The difficulty that was encountered with the radiative models thus persists with the convective models, and one may wonder whether the difficulty still lies with the opacities, this time with $\mathrm{H}, \mathrm{He}$ or with the lower temperature $\mathrm{H}^{-}$and molecular opacities.

\section{Pop. II Cepheid Pulsations}

Pop. II Cepheids are variable stars that have lower metallicity than their classical siblings. They also are believed to have much smaller masses for the same luminosity, which makes them on the one hand much more linearly unstable to vibrations, and on the other hand causes much larger pulsation amplitudes.

${ }^{3}$ If one disregards V440 Per which may be an oddball. 
Observationally, these stars, known as W Vir and RV Tau stars, range from periodic at short periods to strongly irregular at cycling times of $70 \mathrm{~d}$. The irregular behavior seems to set in at period of about 25-30 d (Arp 1955, Pollard et al. 2000).

The recent nonlinear analysis of the AAVSO observational data of $\mathrm{R}$ Sct (Buchler et al. 1995) and of AC Her (Kolláth et al. 1998) showed very clearly that the mechanism for the irregular behavior is the nonlinear interaction between the excited (linearly unstable) mode and a (linearly stable) overtone. Technically speaking, the dynamics take place in a $4 \mathrm{D}$ subspace of phase-space, thus the pulsations are low-dimensional chaos.

This analysis corroborates numerical hydrodynamical results obtained a decade ago which showed that the irregular behavior of $\mathrm{W}$ Vir models was also due to low-dimensional chaos (Buchler \& Kovács 1986; Kovács \& Buchler 1997; Aikawa 1987; Buchler, Goupil, \& Kovács 1987). However, the onset of the irregular behavior occurred in these radiative models with periods as low $8 \mathrm{~d}$, i.e. much lower than observations indicate. Glasner \& Buchler (1990) included a very simplistic MLT model in the hydro-code, and this pushed the onset of chaos to higher periods. More recent calculations with the TC Florida code also show a shift in the same direction.

The basic nature of the irregular behavior is now understood, but clearly more work is necessary to obtain more detailed agreement with the observations.

\section{Mira Pulsations}

Convection plays an essential role in the cool and very extended Mira variables, and they are hard to model with much confidence. There is still a debate about whether the stars pulsate in the fundamental or the first overtone mode.

Ya'ari \& Tuchman (1996) have modeled the nonlinear pulsations of these stars with very interesting results (see also Dorfi, Feuchtinger, \& Gautschy 2000). The large amplitude pulsations that develop cause a structural rearrangement of the star. Consequently the nonlinear period is quite different from both the linear fundamental and first overtone periods. However, convection is treated with a standard time-independent MLT approach, and unfortunately eddy viscosity is ignored in their computations. The latter reduces the pulsational amplitude, and could cause a qualitative change in the results.

\section{Conclusions}

In recent years several groups have included a description of turbulent convection in their hydro-codes. The addition of a simple nonlinear time-dependent diffusion equation for turbulent energy with concomitant convective flux and eddy viscous pressure has led to important improvements in RR Lyrae and Cepheid models. Most striking has been the ability of these codes to model DM pulsations in both RR Lyrae stars and Cepheids.

However, it is clear that small discrepancies remain in the RR Lyrae models, both the single mode RRab and RRc, as well as in the double-mode RRd. The next step is to see if a better calibration of the free $\alpha$ parameters can bring us in a better agreement with the plethora of observational data. 
In the Cepheid modeling, one obtains reasonable agreement for the Galactic Cepheids, even with a preliminary crude calibration, but for the time being it remains a puzzle why the low $Z$ models fail so strikingly.

Acknowledgments. It is a pleasure to acknowledge numerous valuable discussions with my collaborators Z. Kolláth, P. Yecko, M.-J. Goupil, J.-P. Beaulieu and M. Feuchtinger. This work has been supported by the National Science Foundation (AST9528338, AST9819608, INT9820805).

\section{References}

Aikawa, T. 1987, ApSS, 139, 281

Arp, H. C. 1955, AJ, 60, 1

Baker, N. 1987, in Physical Processes in Comets, Stars, and Active Galaxies, ed. W. Hillebrandt, E. Meyer-Hofmeister, \& H.-C. Thomas (New York: Springer), 105

Beaulieu, J. P., Grison, P., Tobin, W., et al. 1995, A\&A, 303, 137

Bono, G. \& Stellingwerf, R. F. 1994, ApJS, 93, 233

Bono, G., Caputo, F., Castellani, V., \& Marconi, M. 1997, A\&A, 121, 327 [BCCM97]

Bono, G., Caputo, F., Castellani, V., \& Marconi, M. 1999, ApJ 512, 711

Buchler, J. R. 1998, in ASP Conf. Ser. Vol. 135, A Half Century of Stellar Pulsation Interpretations: A Tribute to Arthur N. Cox, ed. P. A. Bradley \& J. A. Guzik (San Francisco: ASP), 220

Buchler, J. R. \& Kandrup, H. E. 2000, in Astrophysical Turbulence and Convection, Ann. N. Y. Acad. Sci. (in press)

Buchler, J. R. \& Kolláth, Z. 2000, in Buchler \& Kandrup. loc. cit. [BK00]

Buchler, J. R. \& Kovács, G. 1986, ApJ, 320, L57

Buchler, J. R., Kolláth, Z., Beaulieu, J. P., \& Goupil, M. J. 1996, ApJ, 462, L83

Buchler, J. R., Kolláth, Z., Serre, T., \& Mattei, J. 1996, ApJ, 462, 489

Buchler, J. R., Goupil, M. J., \& Kovács, G. 1987, Physics Lett. A, 126, 177

Buchler, J. R., Serre, T., Kolláth, Z., \& Mattei, J. 1995, PhysRevLetters, 74, 842

Buchler, J. R., Yecko, P., Kolláth, Z., \& Goupil, M. J. 1999, Turbulent Convection in Pulsating Stars, in Giménez et al. loc. cit.; [astro-ph/9901188] [BYKG]

Cattaneo, F., Brummell, N. H., \& Toomre, J. 1991, ApJ, 370, 282

Cox, J. P. \& Giuli R. T. 1969, Principles of Stellar Structure (New York: Gordon and Breach)

Dorfi, E., Feuchtinger, M. U., \& Gautschy, A. 2000, in these proceedings, p. 109

Feuchtinger, M. U. 1998, A\&A, 337, L29

Feuchtinger, M. U. 1999a, A\&AS, 136, 217 [F99a]

Feuchtinger, M. U. 1999b, A\&A, in press [F99b]

Gehmeyr, M. 1992, ApJ, 399, 265

Gehmeyr, M. \& Winkler, K.-H. A. 1992, A\&A, 253, 92; ibid. 253, 101

Gieren, W. P., Fouqué, P., \& Gómez, M. 1997, ApJ, 488, 74 
Giménez, A., Guinan E. F., \& Montesinos, B. (ed.) 1999, ASP Conf. Ser. Vol. 173, Theory and Tests of Convection in Stellar Structure (San Francisco: ASP)

Glasner, A. \& Buchler, J. R. 1990, in NATO ASI Ser. C302, The Numerical Modeling of Nonlinear Stellar Pulsations; Problems and Prospects, ed. J. R. Buchler (Dordrecht: Kluwer), 109

Kolláth, Z. 2000, in these proceedings, p. 356

Kolláth, Z., Beaulieu, J. P., Buchler, J. R., \& Yecko, P. 1998, ApJ, 502, L55 [KBBY]

Kolláth, Z., Buchler, J. R., Serre, T., \& Mattei, J. 1998, A\&A, 329, 147

Kovács, G. \& Buchler, J. R. 1987, ApJ, 324, 971

Kuhfuß, R. 1986, A\&A, 160, 116

Laney, C.D. 1997 in ASP Conf. Ser. Vol. 135, A Half Century of Stellar Pulsation Interpretations, ed. P. A. Bradley \& J. A. Guzik (San Francisco: ASP), 180

Pollard, K. P., Alcock, C., Allsman, R. A., et al. (The MACHO Collaboration) 2000 , in these proceedings, p. 89

Stellingwerf, R. F. 1982, ApJ, 262, 330

Udalski, A., Soszyński, I., Szymański, M., et al. 1999, Acta Astron., 49, 1 [astro-ph/9903391]

Wuchterl, G. \& Feuchtinger, M. 1998, A\&A, 340, 419

Ya'ari, A. \& Tuchman, Y. 1996, ApJ, 456, 350

Yecko, P., Kolláth Z., \& Buchler, J. R. 1998, A\&A, 336, 553 [YKB]

\section{Discussion}

Pawel Moskalik: You have mentioned the possibility of constraining the $M-L$ relation with two resonances. I have done that for the Galactic Cepheids. For $P_{2} / P_{0}=0.5$ at $10 \mathrm{~d}$ and $P_{4} / P_{1}=0.5$ at $4.6 \mathrm{~d}$, and metallicity $Z=0.02, \mathrm{I}$ find that both resonances are simultaneously satisfied for the relation $\log L=$ $3.68 \times \log M+0.65$. The slope has been adopted from the paper of Becker, Iben, $\&$ Tuggle, but the zero point is constrained by the resonances. For a zero point of 0.65 both resonances are reproduced with the same $M-L$ relation.

Harold McNamara: It might be an interesting exercise to apply your theory to $\delta$ Scuti stars to see:

1. why the width of the instability strip for the high amplitude stars is narrow (about $300 \mathrm{~K}$ ); and

2. if you can predict some parameter to distinguish between fundamental mode and first overtone pulsations.

Shashi Kanbur: The Type II Cepheids should also obey a $T_{\min }, T_{\max }$ constraint such that short-period Type II Cepheids have a $T_{\min }$ constraint, and long-period Type II Cepheids have a $T_{\max }$ constraint.

Douglas Gough: It is interesting that you were unable to match your theory to all the observational constraints with a unique set of parameters, even though you 
have seven of them. Perhaps it is because you implicitly set an eighth parameter to zero. Your mixing-length prescription appears to be based on fluxes derived from gradients of the mean state; that is a common outcome of analyses of systems using kinetic theory, which is basically what mixing-length theory is, and is valid provided information travels at a speed much less than the mean speed of the transporting 'particles' - here fluid elements. When approximating the Liouville equation for such particles under such conditions, advection in phase space is fast and one is left with a diffusion equation (whose solutions formally propagate with infinite speed). But, if the finite advection speed is taken into account, an additional term arises in the equation; its relative importance might be scaled by the eighth parameter in the uncertain theory. The importance of this parameter comes about because, by including it, one does not merely distort a function (representing the solution), but one changes the mathematical structure of the governing equations: as the parameter increases from zero, the equations change from diffusion-like to wave-like, and the solutions can be qualitatively different. If, therefore, this phase-space advection is important in Galactic Cepheids, but not in low- $Z$ Cepheids, or vice versa, one would expect that one cannot calibrate a theory to both, if that theory ignores this process. Alternatively, your parameters may not be universal constants, which is, perhaps just as likely.

Giuseppe Bono: Just two comments:

1. I am very much interested in nonlinear calculations you and M. Feuchtinger recently provided. In particular, let me note that the RR Lyrae instability strip predicted by Michael differs by no more than $100-150 \mathrm{~K}$ from our predictions (Bono et al. 1997). This fact is supporting the reliability of nonlinear codes.

2. Fortunately Cepheids present a lot of robust observables, and I think in the near future we should focus on the comparison between the theory and observations to assess the reliability of our physical assumptions. 